\title{
Dissipative Josephson junction of an optical soliton and a surface plasmon
}

\author{
Yasa Ekşioğlu, Özgür E. Müstecaplıŏlu, and Kaan Güven \\ Department of Physics, Koç University, Istanbul 34450, Turkey
}

(Received 4 October 2012; published 20 February 2013)

\begin{abstract}
We examine the dynamics of a dissipative photonic Josephson junction formed by the weak coupling of an optical soliton in a nonlinear dielectric waveguide and a co-propagating surface plasmon along a parallel metal surface with a linear dielectric spacer. We employ a heuristic model with a coupling function that depends on the soliton amplitude and consider two phenomenological dissipation mechanisms separately: angular-velocity dissipation and population imbalance dissipation. In the former dissipation mechanism, the system exhibits a phase-slip phenomenon where the odd- $\pi$ phase modes decay into even- $\pi$ phase modes. The latter damping mechanism sculptures the phase space significantly by introducing complex features, among which, Hopf-type bifurcations are notable. We show that some of the bifurcation points expand to stable limit cycles for certain regimes of the model parameters.
\end{abstract}

DOI: 10.1103/PhysRevA.87.023823

PACS number(s): 42.65.Sf, 42.65.Tg, 03.75.Kk, 03.75.Lm

\section{INTRODUCTION}

As the research on the interaction of light with metallic structures matures as a well-established technology called plasmonics [1-3], the coupling of surface plasmons (SPs) to different light sources is being investigated with the motivation that controlling surface plasmons offers the potential for developing different types of SP-integrated nanophotonic devices [4-6]. In particular, the coupling between SPs and confined light modes is widely investigated. A recent proposal was based on the resonant interaction between the SPs on a metal surface and the co-propagating soliton in a nonlinear dielectric medium [7]. Under a classical formulation, this system exhibits rich nonlinear dynamical features where the interaction depends on the soliton amplitude, as such, it may be utilized to manipulate the SP propagation. As an additional feature, it has been shown [8] that the system is akin to the bosonic Josephson junction (BJJ) of Bose-Einstein condensates [8-12] so that similar and different nonlinear Josephson junction features may be realized in this optic-plasmonic system.

Although this is an exciting analogy, surface plasmons are subject to strong dissipative effects in the host metal, and the perfect Josephson junction is a too-idealized model. In the present paper, we aim to perform a more faithful representation of the system by taking the dissipation into account. Remarkably, we find that the dissipation can bring some benefits if it could be introduced under control. The junction can exhibit stable dynamics despite dissipation. Based on this motivation, here, we investigate the dissipation effects of the coupled soliton-SP system based on the model introduced in Ref. [7] and then formulated as a nonlinear Josephson junction [8]. We note that dissipation effects in the BJJ were previously studied [9], which might be useful to refer to for comparison. We employ a standard dynamical analysis of the system in the phasespace representation and investigate how different dissipation mechanisms and other model parameters affect the phasespace landscape. Bifurcations in the system are highlighted, which may be induced in certain regimes of these parameters.

*yeksioglu@ku.edu.tr

\section{MODEL AND THEORETICAL FORMULATION}

Construction of a classical theoretical model for a coupled soliton-surface-plasmon system has recently been addressed in a number of publications. An elegant initiative was the heuristic model introduced by Bliokh and collaborators [7] in which the interaction between the soliton and the surface plasmon was formulated as a coupled nonlinear-linear oscillator system with the coupling parameter being dependent on the soliton amplitude. Although being constrained by a number of simplifying assumptions, the model predicts stationary coupled modes of the soliton and the surface plasmons under feasible parameter regimes. In another paper, stationary and quasistationary solutions for the so-called soliplasmons were investigated under an asymmetric coupling model [13]. In this paper, we employ the heuristic coupled-oscillator model, albeit with an improved coupling parameter adapted from the recent formulations. Our main motivation is to incorporate the correct behavior for the coupling in the strong soliton and strong surface-plasmon amplitudes, respectively [13]. We begin by recapitulating the heuristic theoretical model in Ref. [7] where the interaction between optical solitons and SPs is discussed in the context of a resonant optical system: A soliton propagating parallel to a metal interface can excite surface plasmons by its evanescent lateral tail, which, in turn, interacts in resonance with the soliton along the propagation. The soliton and the SP are assumed to be co-propagating, hence, only the spatial dynamics of the propagation is of concern. The nonlinearity of the system is assumed to be confined at a distance $d$ from the metal interface such that the surface-plasmon propagation retains linearity, and the coupling between the soliton and the SP fields is weak (see Fig. 1). The formulation is based on a two-dimensional coordinate system in which $y$ is the propagation direction and $x$ is the lateral direction.

The total electric field of the system is introduced by the following variational ansatz in which the soliton and SP fields are written in a product form of their respective longitudinal $\left[c_{p, s}(y)\right]$ and transverse amplitudes:

$$
E(x, y)=c_{p}(y) \psi_{p}(x)+c_{s}(y) \psi_{s}\left(x,\left|c_{s}\right|\right) .
$$

Here, $\psi_{p}=e^{-\kappa_{p} x}$, and $\psi_{s}=\operatorname{sech}\left[\kappa_{s}(x-d)\right]$. Evanescent wave vectors are $\kappa_{p}=\sqrt{k_{p}^{2}-k^{2}}$ and $\kappa_{s}=k \sqrt{\gamma / 2}\left|c_{s}\right| . k$ 


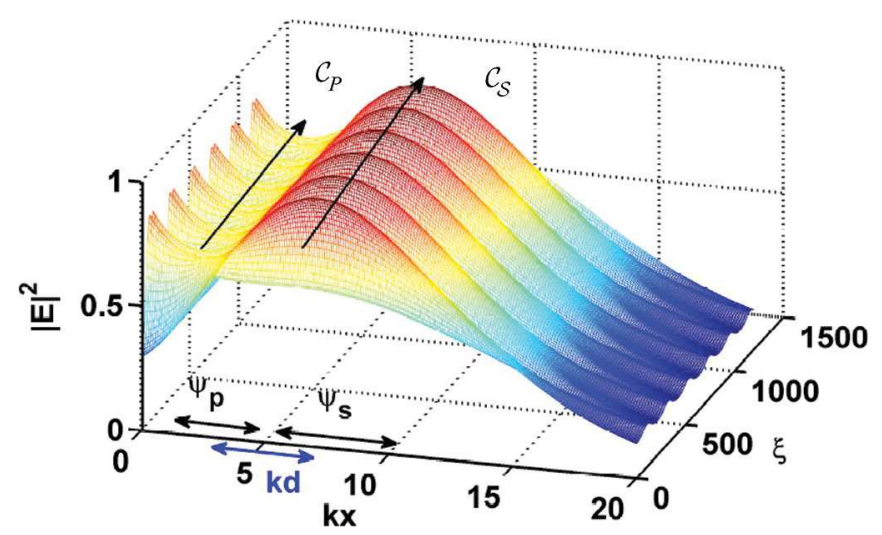

FIG. 1. (Color online) Schematic of the coupled surface-plasmon optical soliton model. $|E|^{2}$ vs transverse profile dimensionless direction $k x$ and dimensionless propagation direction $\xi ; k d$ is the dimensionless distance between the metal interface and the dielectric nonlinearity. All parameters are dimensionless.

is the incoming wave vector, whereas, $k_{p}$ is the SP wave vector. $\gamma$ is the nonlinearity parameter of the dielectric strip. The longitudinal amplitudes $c_{p, s}(y)$ obey the coupled spatial propagation wave equations,

$$
\ddot{c}_{p}+\beta_{p}^{2} c_{p}=q\left(\left|c_{s}\right|\right) c_{s}, \quad \ddot{c}_{s}+\beta_{s}^{2} c_{s}=q\left(\left|c_{s}\right|\right) c_{p} .
$$

where $\ddot{c}_{p, s} \equiv \frac{\partial^{2} c_{p, s}}{\partial \xi^{2}}$ and $\xi \equiv k y$ is the dimensionless propagation coordinate. $\beta_{p}=k_{p} / k$ and $\beta_{s}=1+\gamma\left|c_{s}\right|^{2} / 4$ are the propagation constants of the SP and soliton, respectively. $q\left(\left|c_{s}\right|\right)$ is the coupling function, which we discuss further below. The coupled equations are linearized by introducing $c_{p, s}=\mathcal{C}_{p, s} e^{i \xi}$ into Eq. (2) and by employing the slowly varying amplitude approximation to discard higher-order derivatives. The final set of equations is then

$$
\begin{gathered}
-i \dot{\mathcal{C}}_{p}=v_{p} \mathcal{C}_{p}-\frac{q\left(\left|\mathcal{C}_{s}\right|\right)}{2} \mathcal{C}_{s}, \\
-i \dot{\mathcal{C}}_{s}=-\frac{q\left(\left|\mathcal{C}_{s}\right|\right)}{2} \mathcal{C}_{p}+v_{s}\left(\left|\mathcal{C}_{s}\right|\right) \mathcal{C}_{s} .
\end{gathered}
$$

$v_{p} \equiv \beta_{p}-1 \ll 1, v_{s} \equiv \beta_{s}-1 \ll 1$ are the small deviations of the dimensionless propagation constants of the surface plasmon and soliton, respectively.

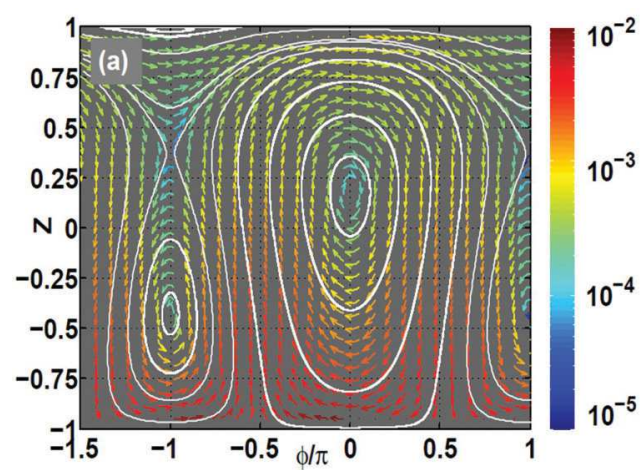

Evidently, the coupling function plays the main role in these equations. Qualitatively, it should describe the interaction through the overlap of the evanescent tails of the soliton and plasmon in the (linear) dielectric spacer between the metal interface and the nonlinear dielectric waveguide. Since the lateral profile of a soliton depends on its amplitude, the coupling function is expected to exhibit this dependence too. An analytical formulation of the coupling function was provided in Ref. [13] with the remark that the equation system was not symmetric in the coupling function in general. Within the heuristic model, the following functional form captures the expected behavior in the strong soliton and strong SP amplitude limits:

$$
q\left(\left|\mathcal{C}_{s}\right|\right) \simeq\left|\mathcal{C}_{S}\right| e^{-\sigma k d \sqrt{\gamma / 2}\left|\mathcal{C}_{s}\right|}
$$

Here, $\sigma$ is an overall constant which we introduce as a parameter to account for the other constants (e.g., permittivities and propagation constants). The key parameters are preserved in the functional form. In the strong soliton amplitudes $\left(\left|\mathcal{C}_{s}\right| \approx\right.$ $1)$, the exponentially decaying term dominates the coupling. In the weak soliton amplitude, the coupling is proportional to $\left|\mathcal{C}_{s}\right|$ since the soliton lateral profile in Eq. (1) would be wider, resulting in a larger overlap.

The analogy between this model and the BJJ dynamics $[10,12]$ is revealed when we further substitute $\mathcal{C}_{s, p}=C_{s, p} e^{i \phi_{s, p}}$ into Eq. (4) and introduce a new variable set consisting of the fractional population imbalance $Z=\left(\left|C_{s}\right|^{2}-\left|C_{p}\right|^{2}\right) / N$, and the relative phase difference between the soliton and the SP is $\phi=\phi_{s}-\phi_{p} ; N=\left(\left|C_{s}\right|^{2}+\left|C_{p}\right|^{2}\right) \equiv 1$ is normalized as a constant for the isolated system, and the total energy is conserved under the optical soliton-surface-plasmon interaction $[7,8]$,

$$
\begin{gathered}
\dot{Z}=-q(Z) \sqrt{1-Z^{2}} \sin \phi, \\
\dot{\phi}=\Lambda Z+\Delta E+\frac{q(Z) Z}{\sqrt{1-Z^{2}}} \cos \phi,
\end{gathered}
$$

where $\Lambda=\frac{\gamma N}{8}$ is the nonlinearity parameter and $\Delta E \equiv \Lambda-$ $v_{p}$ parametrizes the asymmetry between the soliton and the SP states (similar to the asymmetry between the wells of a

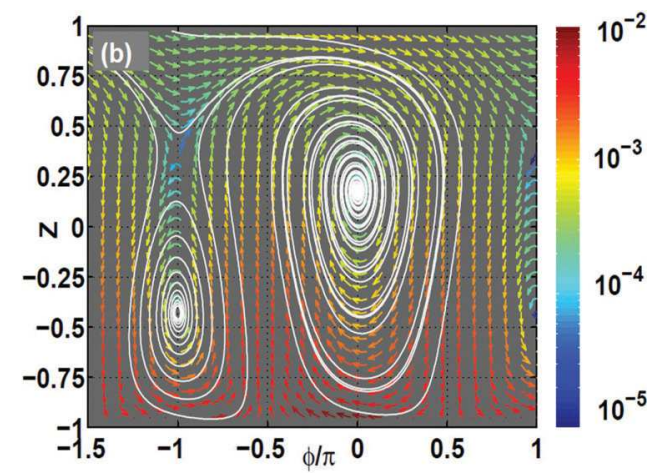

FIG. 2. (Color online) (a) The $Z$ - $\phi$ phase space of the dissipationless SP-soliton coupled system with $k d=6, \Lambda=0.01$, and $\Delta E=-0.0025$. The magnitude of the gradient plot is color coded logarithmically. White curves show typical open and closed trajectories, (b) phase-space trajectory with parameters of (a) and with angular-velocity-dependent dissipation $\eta=0.2$. Phase slip occurs between the $\phi=-\pi$ and the $\phi=0$ modes. All parameters are dimensionless. 

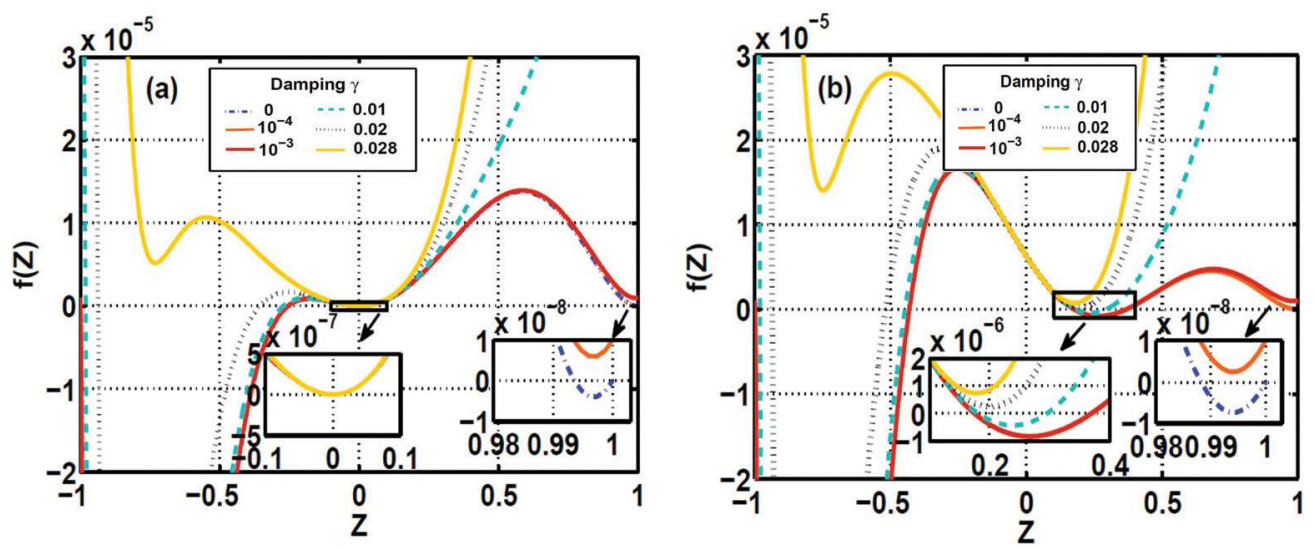

FIG. 3. (Color online) (a) Plot of Eq. (13) for $k d=6, \Lambda=0.01$, and $\Delta E=0$ at various values of the dissipation constant $\zeta$. Roots of $f(Z)$ are the critical points in the phase space, (b) plot of Eq. (13) for $k d=6, \Lambda=0.01$, and $\Delta E=-0.0025$ at various values of the dissipation constant $\zeta$. Roots of $f(Z)$ are the critical points in the phase space. All parameters are dimensionless.

double-well system). The coupling function $q$ takes the form

$$
q(Z) \simeq \sqrt{\frac{(1+Z)}{2}} e^{-\sigma k d \sqrt{2 \Lambda(1+Z)}} .
$$

We stress that the $Z$ dependence makes an inherently dynamic coupling as opposed to the constant (or externally tunable) coupling parameter present in BJJ systems. With this formulation, the coupled soliton-SP system can be described as a nonlinear Josephson junction. In the calculations presented below, we fix the dimensionless separation parameter $k d=6$, which is the same as the resonant coupling of the optical soliton and surface-plasmon system $\left(\left|C_{s}\right| \sim\left|C_{p}\right|, q \ll 1\right)$ [7], and the range of the model parameters is chosen to be around the resonant coupling regime between $\Lambda \simeq 0.003-0.03$ and $\Delta E \simeq-0.4-0.4$, accordingly. Evidently, using larger $k d$ values would confine the interaction to smaller soliton amplitudes and, eventually, would result in the decoupling of the soliton and the SP completely. Small $k d$ values would not be applicable in the weak-coupling approximation.

\section{ANGULAR-VELOCITY-DEPENDENT DISSIPATION}

Owing to the analogy to the BJJ models [9], the first dissipation mechanism we consider is about the incoherent exchange of photons between the soliton and the SP. This is introduced by the term $\eta \dot{\phi}$ in the following equations:

$$
\begin{gathered}
\dot{Z}=-q(Z) \sqrt{1-Z^{2}} \sin \phi-\eta \dot{\phi}, \\
\dot{\phi}=\Lambda Z+\Delta E+\frac{q(Z) Z}{\sqrt{1-Z^{2}}} \cos \phi,
\end{gathered}
$$

Under coordinate reversal $\xi \rightarrow-\xi$, we have $Z \rightarrow Z$ and $\phi \rightarrow-\phi$, hence, the dissipative term $\eta \dot{\phi}$, appearing in the equation for $\dot{Z}$, represents an irreversible process correctly. The phase space of the dissipationless and dissipative system is shown in Figs. 2(a) and 2(b), respectively. The gradient distribution is given with color-coded magnitudes, and typical trajectories are indicated by white curves. The phase space is characterized by the zero-(even-) phase and $\pi$ - (odd-) phase modes where the critical points are located. There is always one stable point at $\phi=0$. At $\phi=\pi$, one (stable) to three (two stable, one saddle) critical points may emerge depending on the values of the model parameters $\Lambda, k d$, and $\Delta E$ [8]. In Fig. 2(a), sample trajectories (white curves) show anharmonic closed orbits around stable critical points and open trajectories. The color indicates that the gradients get steeper in the SPdominant population imbalance region $(Z<0)$.

Since the critical points are determined by the $\dot{Z}=0, \dot{\phi}=$ 0 condition, the $\dot{\phi}$-dependent dissipation does not alter the location of the critical points in the phase space, but the eigenvalues of the Jacobian acquire nonzero real parts such that the stable critical points at $\phi=\pi$ become the source and the critical point at $\phi=0$ becomes a sink. While the trajectories flow towards the sink points, the phase-slip phenomenon is observed from $\phi=$ odd- $\pi$ modes to $\phi=$ even- $\pi$ modes as reported for the BJJ systems [9]. Depending on the value of the asymmetry parameter $\Delta E$, the phase slip occurs with a nonzero change in the averaged population imbalance $\langle Z\rangle$.

With a larger (smaller) dissipation constant, the typical phase diagram depicted in Fig. 2(b) gets compressed (extended) along the phase axis, but the features described above remain the same. Thus, whereas, the transient behavior depends on $\eta$, the final state of the system is independent: $\phi_{s}=\phi_{p}$ and a fixed value of $Z$.

\section{POPULATION IMBALANCE DISSIPATION}

In the following, we introduce the set of equations that incorporates a dissipation proportional to the population imbalance $Z$. In the context of BJJ systems, such a dissipation arises from the finite lifetime of excited states of two condensates in different hyperfine levels in a single harmonic

TABLE I. Classification of fixed points at different dissipation regimes for Fig. 3(b), sso: spiral source; ssi: spiral sink.

\begin{tabular}{lcccccc}
\hline \hline$\zeta$ & I & II & III & IV & V & VI \\
\hline $0<\zeta<8.5 \times 10^{-5}$ & Saddle & sso & ssi & Saddle & ssi & Saddle \\
$8.5 \times 10^{-5} \leqslant \zeta<0.016$ & Saddle & sso & ssi & Saddle & & \\
$0.016 \leqslant \zeta<0.027$ & Saddle & sso & & & & \\
$0.027 \leqslant \zeta$ & & & & & & \\
\hline \hline
\end{tabular}



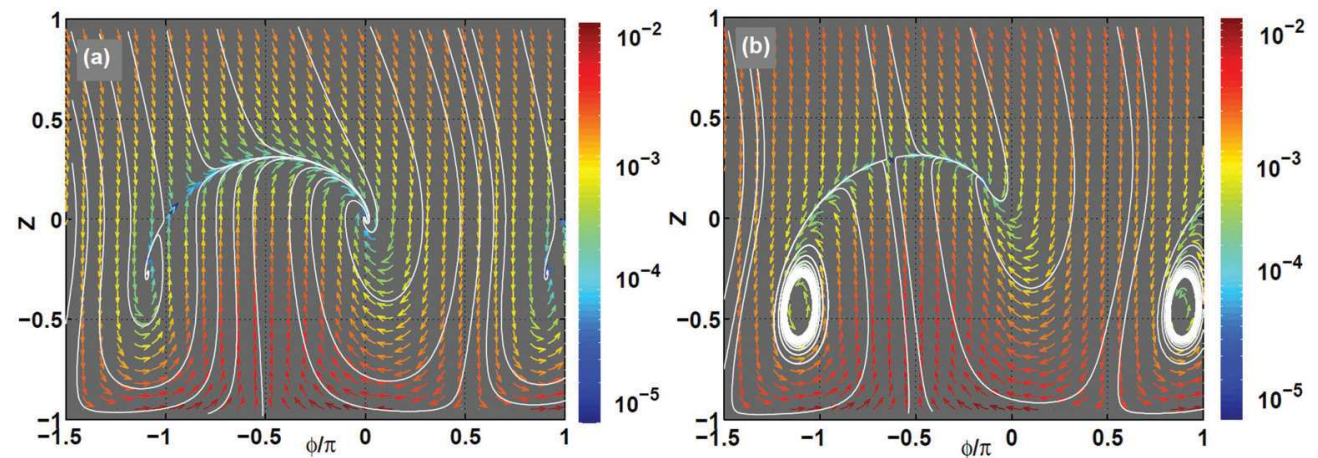

FIG. 4. (Color online) Phase-space plot of a coupled soliton-SP system with population imbalance dissipation. Model parameters are $k d=$ $6, \Lambda=0.01$, and $\zeta=0.01$. All parameters are dimensionless. (a) $\Delta E=0$ and (b) $\Delta E=-0.0025$. White curves indicate sample trajectories. The magnitude of the gradient plot is color coded logarithmically. In (b), only trajectories exterior to the limit cycles are shown for clarity.

trap connected by tunneling transitions [14]. In the analogous mechanical system of a momentum-shortened pendulum, this corresponds to a damping proportional to the angular momentum variable [15], which we consider to be applicable to the soliton-SP system phenomenologically,

$$
\begin{aligned}
& \dot{Z}=-q(Z) \sqrt{1-Z^{2}} \sin \phi-\zeta Z, \\
& \dot{\phi}=\Lambda Z+\Delta E+\frac{q(Z) Z}{\sqrt{1-Z^{2}}} \cos \phi .
\end{aligned}
$$

The first thing we note here is that this dissipation term can modify the critical points in the phase space significantly: their existence, location, and corresponding Jacobian eigenvalues. For determining the critical points using $\dot{Z}=0, \dot{\phi}=0$, and the identity $\sin ^{2}(\phi)+\cos ^{2}(\phi)=1$, we obtain the following root equation in $Z$ :

$$
Z^{4} \zeta^{2}+\left(1-Z^{2}\right)\left[(\Delta E+\Lambda Z)^{2}\left(1-Z^{2}\right)-q(Z)^{2} Z^{2}\right]=0 .
$$

Figure 3 is a plot of this equation for fixed $k d=6, \Lambda=0.01$, and for (a) $\Delta E=0$ and (b) $\Delta E=-0.0025$ with several orders of magnitude of the dissipation constant $\zeta$. The dasheddot (blue) curve is plotted for the dissipationless case $(\zeta=0)$ for reference. For $\Delta E=0, Z=0$ is always a critical point as all curves in Fig. 3(a) are tangent to $Z=0$ at this point, irrespective of dissipation. This can also be inferred from
Eq. (13). Critical points close to $Z \approx 1$ are most sensitive to the dissipation strength, and they already disappear at weak dissipation $\left(\zeta \approx 10^{-4}\right)$. For $\Delta E=-0.0025$, as the dissipation gets stronger, the critical points collide and annihilate in pairs. This is actually indicating the presence of saddle-node-type bifurcations in the system, which we show further below. Finally, above $\zeta=0.0275$, no critical point is present in the phase space. The sensitivity of the critical points to the dissipation strength increases rapidly after $\zeta>0.01$. Table I classifies the critical points at different dissipation regimes for $\Delta E=-0.0025$. Complex eigenvalues of the Jacobian with positive (negative) real parts define spiral source (sink) points, and the saddles have zero eigenvalues.

According to the typical ranges of the dissipation parameter, we now investigate the behavior of the system in the phase space. Figure 4(a) is plotted for $k d=6, \Lambda=0.01, \Delta E=0$, and $\zeta=10^{-2}$. These values correspond to the dashed (cyan) curve in Fig. 3(b). $Z=0$ is a spiral sink at $\phi=0$ and a saddle point at $\phi=\pi$. Another spiral sink is located at $Z=-0.28, \phi=0.85 \pi$. The saddle point at $Z=-0.98, \phi=$ $0.5 \pi$ is not visible at this scale.

In Fig. 4(b), the same parameters as in Fig. 4(a) are used, but $\Delta E=-0.0025$. Remarkably, a stable limit cycle is present, indicating the existence of bifurcations induced by the model parameters. In Fig. 5(a), the dissipation constant is increased to $\zeta=0.02$ for which a larger limit cycle is observable. Finally,
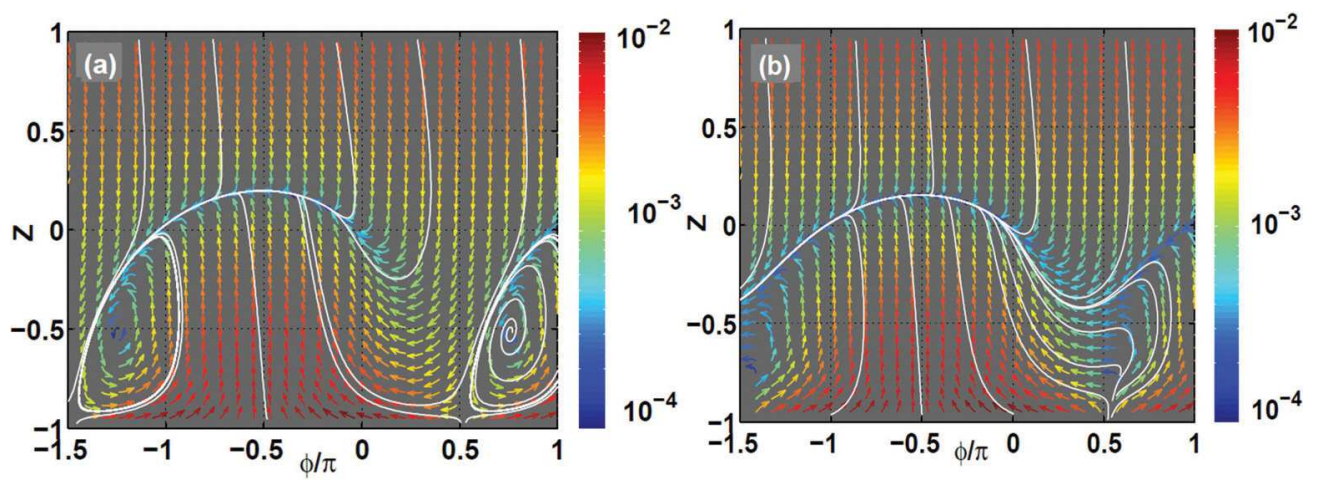

FIG. 5. (Color online) Phase-space plot of the coupled soliton-SP system with population imbalance dissipation. Model parameters are $k d=6, \Lambda=0.01$, and $\Delta E=-0.0025$. All parameters are dimensionless. (a) $\zeta=0.02$. Exterior and interior trajectories shown for left and right limit cycles, respectively. (b) $\zeta=0.028$. White curves indicate trajectories. The gradient plot is color coded logarithmically 

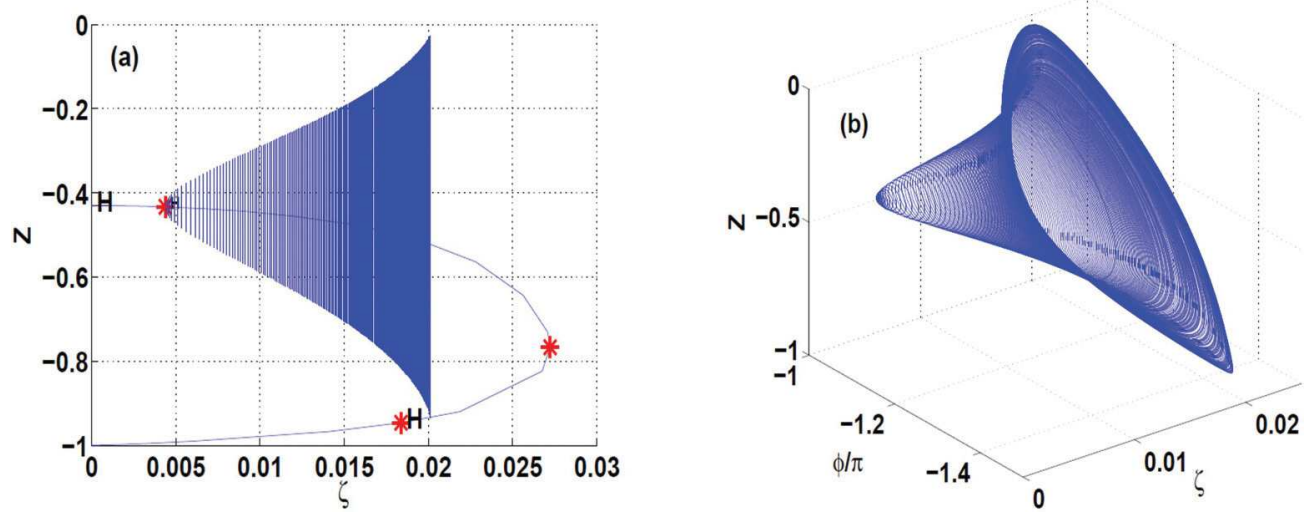

FIG. 6. (Color online) (a) Continuation of the stable point as a function of the dissipation constant. Model parameters are $k d=6, \Lambda=0.01$, and $\Delta E=-0.0025$. All parameters are dimensionless. Andronov-Hopf $(\mathrm{H})$ bifurcation and neutral saddle point are indicated. Unlabeled points correspond to LPs. Vertical lines indicate the extent of the limit cycles along the $Z$ axis. A limit point cycle terminates the limit cycles at $\zeta \approx 2.01$, (b) limit cycles plotted in the $Z-\phi-\zeta$ parametric phase space.

$\zeta \approx 0.028$ is the onset of the regime in which all trajectories flow into a unique stable cycle, and there are no critical points in the phase space. This behavior is similar to that of a Josephson junction between two superconductors with the bias current above the critical current $I \equiv \frac{I_{B}}{I_{C}}>1$ (see, e.g., Strogatz [16]). There, the current phase $\phi$ and angular velocity $\dot{\phi} \equiv y$ satisfy the dimensionless equations,

$$
\begin{gathered}
\dot{\phi}=y, \\
\dot{y}=I-\sin (\phi)-\alpha y .
\end{gathered}
$$

where $\alpha$ is the damping parameter. Note that, for $I>1$, there are no critical points.

\section{A. Codimension-1 bifurcations}

In order to investigate the bifurcation dynamics, we first consider codimension-1 bifurcations, which can be induced by varying a single parameter of the model. We employ a numerical continuation software called MATCONT [17], which computes how a critical point advances in the phase space by varying model parameters and characterizes the point along the continuation according to its Jacobian. We begin by the continuation with respect to the dissipation constant $\zeta$. The fixed parameters are $k d=6, \Lambda=0.01$, and $\Delta E=-0.0025$. Figure 6(a) shows the computed continuation curve on which an Andronov-Hopf $(\mathrm{H})$ bifurcation, limit point (LP), and neutral saddle point (NS) are observed. Andronov-Hopf $(\mathrm{H})$ bifurcation is the birth of a limit cycle from equilibrium, when the equilibrium changes stability via a pair of purely imaginary eigenvalues. The bifurcation can be supercritical or subcritical,

TABLE II. Bifurcation points shown in Fig. 6(a), LP: limit point (fold); H: Andronov-Hopf; NS: neutral saddle point; $a$ is the normal form coefficient for LP and the first Lyapunov coefficient for $\mathrm{H}$.

\begin{tabular}{lcccc}
\hline \hline Label & $\Delta E$ & $\phi^{*}$ & $Z^{*}$ & $a$ \\
\hline $\mathrm{H}$ & 0.0437 & -1.05 & -0.434 & -0.0126 \\
$\mathrm{LP}$ & 0.0272 & -1.42 & -0.767 & -0.109 \\
$\mathrm{NS}$ & 0.0184 & -1.48 & -0.495 & \\
\hline \hline
\end{tabular}

resulting in stable or unstable limit cycle, respectively. At limit point (LP) give rise to limit point bifurcations when the critical equilibrium has one zero eigenvalue. The neutral saddle points (NS) occur when the algebraic sum of two eigenvalues is zero. In Table II, these points are characterized with the normal form coefficient. The Andronov-Hopf bifurcation is supercritical since the first Lyapunov coefficient is negative $[16,18,19]$. The vertical lines indicate the span of limit cycles in the $Z$ axis, emanating from the Hopf point. Figure 6(b) shows the limit cycles in the $Z-\phi-\zeta$ plane. Interestingly, the limit cycles get bigger in the $\phi-Z$ plane for stronger dissipation before they are terminated by a limit point cycle. For reference, the limit cycles in Figs. 4 and 5 are in the range of Fig. 6(b).

Next, the continuation is performed in the asymmetry parameter $\Delta E$. Figures 7(a) and 7(b) show these results for $\zeta=$ 0.0095. In Table III, the bifurcation points are characterized, and the respective normal form coefficients are listed. The first Lyapunov coefficient is negative for the Andronov-Hopf points, which indicates supercritical bifurcation. For clarity, the limit cycles are plotted only on the $\Delta E>0$ side; they are spawned between the two Andronov-Hopf points on the $\Delta E>0$ side too.

In order to gain further insight about the bifurcation, we investigate the continuation curve in the $Z-\Delta E$ plane for different values of $\Lambda$ in Fig. 8 and for $\zeta$ in Fig. 9.

TABLE III. Bifurcation points shown in Fig. 7(a), LP: limit point (fold); H: Hopf point; $a$ is the normal form coefficient for LP and the first Lyapunov coefficient for $\mathrm{H}$.

\begin{tabular}{lcccc}
\hline \hline Label & $\Delta E$ & $\phi^{*}$ & $Z^{*}$ & $a$ \\
\hline LP & -0.340 & 0.878 & -0.997 & 3.917 \\
H & -0.148 & 0.984 & -0.946 & -0.00626 \\
H & -0.00257 & 0.990 & -0.434 & -0.00275 \\
LP & 0.00319 & -1.01 & -0.139 & 0.219 \\
LP & 0.00909 & -0.311 & 0.797 & -0.0148 \\
H & 0.0112 & 0.0102 & -0.4336 & -0.00098 \\
H & 0.167 & 0.0165 & -0.9461 & -0.0623 \\
LP & 0.360 & 0.0102 & -0.434 & -3.92 \\
\hline \hline
\end{tabular}



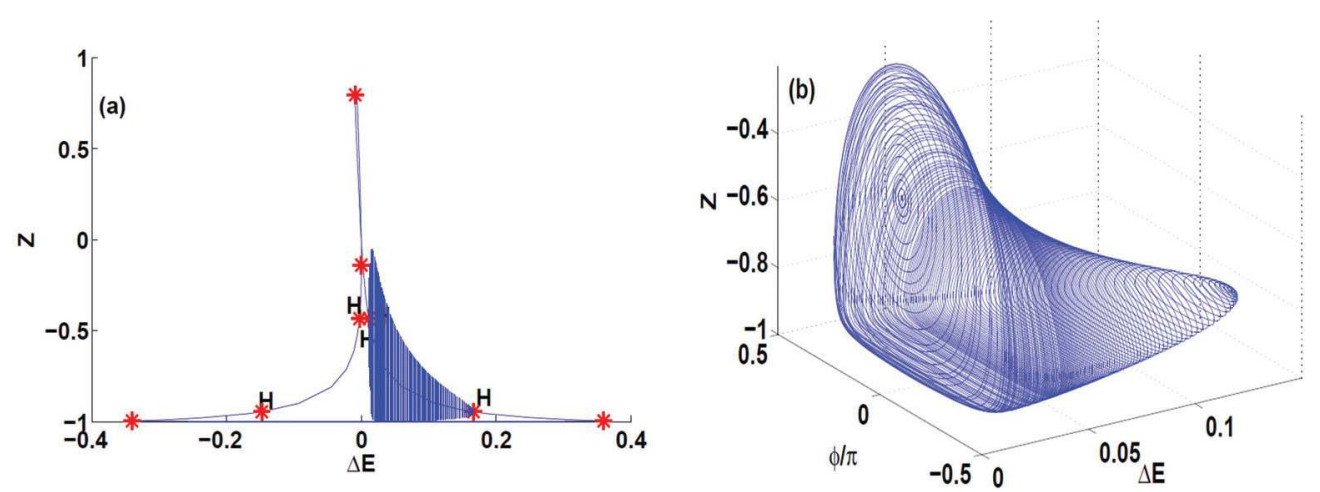

FIG. 7. (Color online) (a) Continuation of the stable point as a function of $\Delta E$. Model parameters are $k d=6, \Lambda=0.01$, and $\zeta=0.0095$. All parameters are dimensionless. Supercritical limit cycles are spawned between the two Hopf bifurcation points. Hopf (H) bifurcations are labeled, and unlabeled points correspond to LPs, (b) visualization of the limit cycles indicated in (a) in the $Z-\phi-\Delta E$ parametric phase space.

Equilibrium points are periodic in the $Z-\phi$ plane, thus, each continuation curve closes onto itself in these parametric planes. The continuation curves extend in a finite region of the $\Delta E-Z$ plane where the range also depends on other model parameters.

In Fig. 8, we depict the effect of nonlinearity $(\Lambda)$ on the continuation curve. In view of Eq. (8), we note that, for a given soliton amplitude, increasing nonlinearity confines the soliton more tightly around its propagation axis and decreases the coupling between the soliton and the SP. Thus, while the continuation curve extends in the whole $Z$ axis for small $\Lambda$ (blue curve), it shrinks and gets confined to the range close to $Z=-1$ for strong nonlinearity (black curve). Note that multiple equilibria are present for a given $\Delta E$ value when $|\Delta E|$ is small (e.g., <0.05). In addition, the nonlinearity also induces different bifurcation points in the continuation curve. For $\Lambda=$ 0.003 , only LPs occur. For $\Lambda=0.03$, LP and H bifurcations are present, but the first Lyapunov exponent is positive (i.e., subcritical Hopf bifurcations). For the intermediate values between these extremes, we observe that the continuation curve hosts multiple $\mathrm{H}$ points, which appear in pairs on the negative and positive sides of the $\Delta E$ axis. These are supercritical, and as shown in Fig. 7, stable limit cycles are spawned between them.

When the dissipation strength $\zeta$ increases, the continuation curve in the $\Delta E-Z$ plane shrinks as shown in Fig. 9. Limit

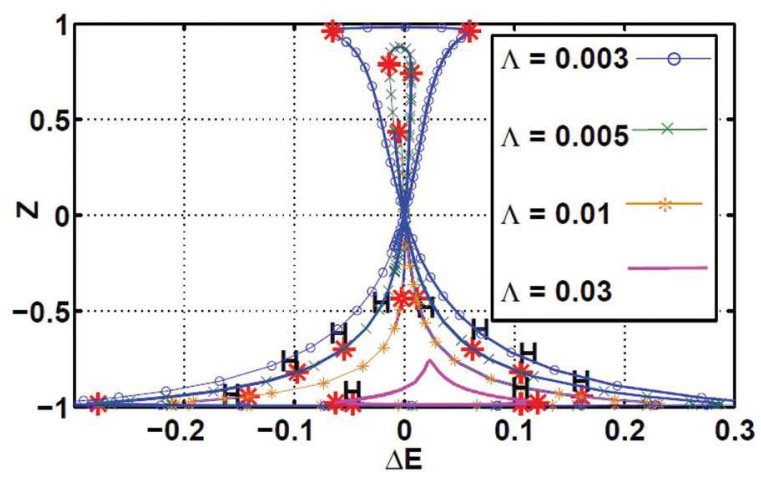

FIG. 8. (Color online) $\Delta E$ continuation of the stable point for different values of $\Lambda$ with $k d=6$ and $\zeta=0.005$. All parameters are dimensionless. Hopf $(\mathrm{H})$ bifurcations are labeled on the continuation curves, and unlabeled points correspond to LPs. point and Hopf bifurcations decorate the continuation curves. The Hopf points close to $Z=-1$ are neutral saddle points. The other Hopf points are supercritical. As shown in Fig. 6, stable limit cycles bifurcate from these Hopf points. Although not shown here, the curve completely disappears for $\zeta>0.028$ since no critical point is present.

We recall that $\Delta E=\Lambda-v_{p}$ is the difference between the (soliton-characteristic) nonlinearity and the (SP-characteristic) propagation constant. Although not obvious in these terms, it is analogous to the asymmetry between the wells of the doublewell potential in BJJ systems and, thus, sizes the asymmetry between the soliton state and the SP state of the Josephson junction. In Figs. 8 and 9, we observe that, for large magnitudes of $\Delta E$ (i.e., irrespective of its sign), the equilibrium points lie in the SP-dominant $(-1<Z \ll 0)$ region. Soliton-dominant equilibrium points occur under weak nonlinearity and weak dissipative conditions only.

\section{B. Codimension-2 bifurcations}

Codimension- 2 bifurcations require the simultaneous variation in two parameters. The blue curve in Fig. 10 shows the

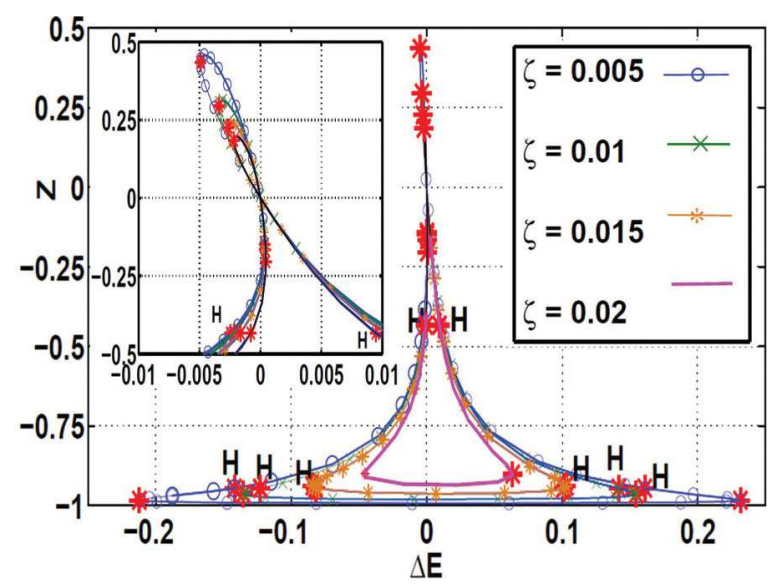

FIG. 9. (Color online) $\Delta E$ continuation of the stable point for different values of $\zeta$ with $k d=6$ and $\Lambda=0.01$. All parameters are dimensionless. Hopf $(\mathrm{H})$ bifurcations are labeled on the continuation curves, and unlabeled points correspond to LPs. The blue curve corresponds to the yellow curve in Fig. 8. 


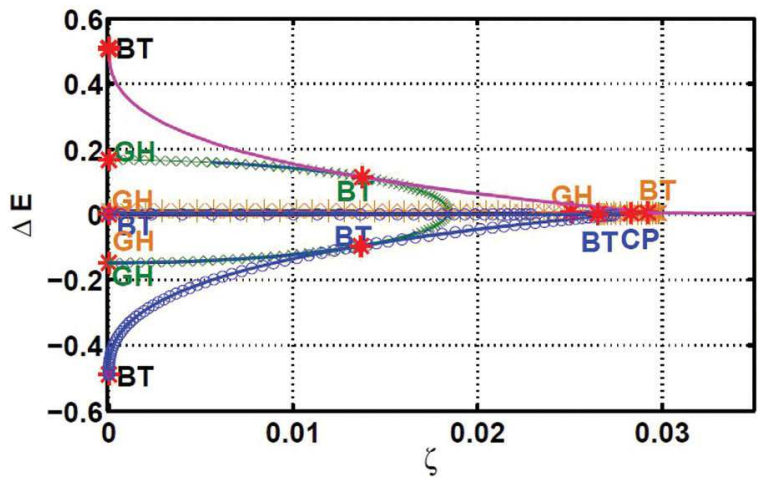

FIG. 10. (Color online) Codimension-2 bifurcation diagram in the $\zeta-\Delta E$ plane. All parameters are dimensionless. Model parameters are $k d=6$ and $\Lambda=0.01$. The circle (blue) curve shows the continuation of an equilibrium point. Asterisks (orange) and cross (green) curves are the Hopf continuation curves from corresponding BT points. The solid (magenta) curve is the limit point continuation curve. BT: Bogdanov-Takens point; CP: cusp point. GH: generalized Hopf point.

continuation of an equilibrium point in the $\zeta-\Delta E$ parameter space. We observe three Bogdanov-Takens (BT) bifurcation points and a cusp point (CP). BT bifurcation implies the existence of three codimension-1 bifurcations nearby, namely, a saddle-node bifurcation, an Andronov-Hopf bifurcation, and a homoclinic bifurcation. From the BT points, the continuation curves of the Hopf point and limit point, respectively, can be obtained. The asterisks (orange) curve are the Hopf curve passing through the BT point close to the cusp point. The cross (green) curve is the Hopf curve passing through the lower BT point. Each Hopf curve has two generalized Hopf points when they intersect the $\zeta=0$ axis. The solid (magenta) curve is the continuation of the limit point from the upper BT point on the cross (green) Hopf curve.

This codimension-2 diagram indicates the regions of $\zeta$ and $\Delta E$ in which stable limit cycles can be generated in the system (see Figs. 10 and 11). In Fig. 7, it was shown that, for fixed $\zeta$, stable limit cycles were spawned between two Hopf points. Here, this corresponds to the region between the asterisks (orange) and the cross (green) curves. The cusp point on the circle (blue) curve marks the value of $\zeta=0.028$ beyond which there are no critical points for $\Lambda=0.1$ and $\Delta E=-0.0025$ as shown in Figs. 3(b) and 5(b). In Table IV, the coordinates of the bifurcation points on the circle (blue) curve are given in the $\zeta-\Delta E$ plane and $\phi-Z$ plane, and their normal form coefficients are listed. BT points have two normal

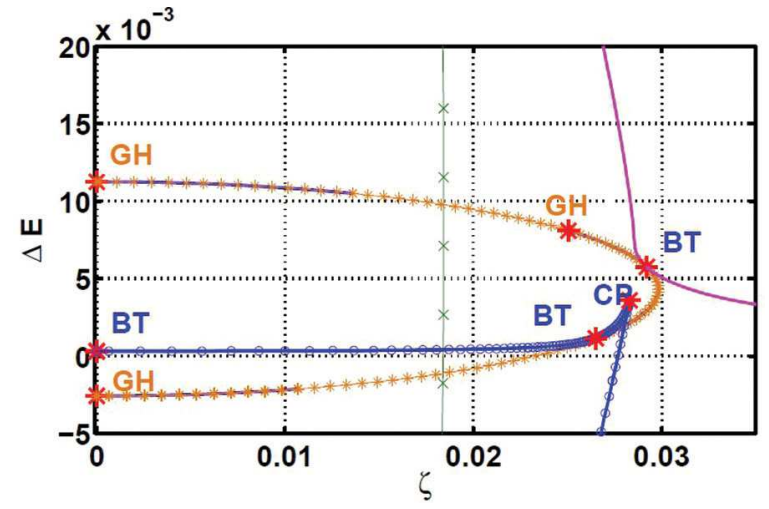

FIG. 11. (Color online) The zoomed view of Fig. 10 around the $\Delta E=0$ axis.

form coefficients with respect to two varying parameters. The cusp point is characterized by a single normal form coefficient.

\section{CONCLUSION}

In this paper, we investigated the dynamical features of a dissipative nonlinear Josephson junction, which was formed by a paraxial optical soliton-surface-plasmon-coupled system. The most notable part of the heuristic model implemented here is the nonlinear nature of the coupling mechanism. For a dissipation proportional to the angular velocity, the system decays into stable fixed points with a constant relative phase and a constant average population imbalance. A particular feature observed here is the phase-slip phenomenon where the odd- $\pi$ modes of the dissipationless system decay into even $-\pi$ modes. For dissipation proportional to the population imbalance, supercritical Andronov-Hopf bifurcation can occur, which is notable in that stable oscillatory modes between the soliton and the surface-plasmon fields can be maintained against strong dissipation. The heavily damped limit resembles the behavior of superconducting Josephson junctions in which the system decays rapidly into a unique oscillating state. In view of this analysis, we propose that the dissipative Josephson junction between the optical soliton and the surface plasmons can exhibit interesting dynamical features, which may be exploited further, based on more rigorous theoretical models.

Finally, we comment that some typical parameter values may serve as guidance for the experimental realization of the system. For the infrared regime, the chalcogenide [20] glasses can provide the medium for soliton propagation [21]. Their transmission band is $0.7-10 \mu \mathrm{m}$, and their linear

TABLE IV. Codimension-2 bifurcations in the $\zeta-\Delta E$ parameter space (blue curve in Fig. 10), BT: BogdanovTakens point; CP: cusp point.

\begin{tabular}{lccccc}
\hline \hline Label & $\zeta$ & $\Delta E$ & $\phi^{*}$ & $Z^{*}$ & $(a, b)^{\mathrm{a}}, c^{\mathrm{b}}$ \\
\hline BT & 0 & $3.19 \times 10^{-4}$ & -1.00 & -0.139 & $\left(1.51 \times 10^{-4},-1.46 \times 10^{-7}\right)$ \\
BT & 0.026 & $1.16 \times 10^{-3}$ & -1.35 & -0.434 & $\left(1.38 \times 10^{-4},-7.18 \times 10^{-2}\right)$ \\
CP & 0.028 & $3.61 \times 10^{-3}$ & -1.46 & -0.65 & -0.10 \\
BT & 0.014 & -0.096 & -1.27 & -0.946 & $(-0.12,0.29)$ \\
\hline \hline
\end{tabular}

a Normal form coefficients of BT.

${ }^{\mathrm{b}}$ Normal form coefficient of CP. 
dielectric constant is around $\epsilon_{d} \sim 5-8$. The nonlinear index of refraction is $n_{2}=10^{-16} \mathrm{~m}^{2} / \mathrm{W}$. The nonlinearity parameter $\gamma$ and nonlinear index of refraction are related as $\gamma=$ $\left(n_{2} k\right) / A_{\text {eff }}$, where $A_{\text {eff }}$ is the effective core area (of the fiber). The nonlinearity parameter for most typical fibers has a value of $\gamma \sim 10^{-3} \mathrm{~W}^{-1} / \mathrm{m}$, but for highly nonlinear fibers, $\gamma \sim 10^{-2} \mathrm{~W}^{-1} / \mathrm{m}$ is achievable [22]. Surface plasmons are typically studied on gold or silver surfaces [23]. Depending on the lateral extent of the evanescent surface-plasmon and soliton fields, the $k d$ for the weak-coupling regime would typically be around $k d \sim 10-100$ [7].

\section{ACKNOWLEDGMENTS}

We acknowledge support by the Science and Technology Research Council of Turkey (TUBITAK) under Project No. 111T285. K. Güven acknowledges partial support from the Turkish Academy of Sciences.
[1] A. V. Zayats, I. I. Smolyaninov, and A. A. Maradudin, Phys. Rep. 408, 131 (2005).

[2] S. A. Maier, Plasmonics: Fundamentals and Applications (Springer, New York, 2007).

[3] A. R. Davoyan, I. V. Shadrivov, and Y. S. Kivshar, Opt. Express 17, 21732 (2009).

[4] Surface Plasmon Nanophotonics, edited by M. L. Brongersma and P. Kik (Springer, Netherlands, 2007).

[5] R. H. Ritchie, Phys. Rev. 106, 874 (1957).

[6] K. Y. Bliokh, Y. P. Bliokh, V. Freilikher, S. Savelev, and F. Fori, Rev. Mod. Phys. 80, 1201 (2008).

[7] K. Y. Bliokh, Y. P. Bliokh, and A. Ferrando, Phys. Rev. A 79, 041803R (2009).

[8] Y. Ekşioğlu, Ö. E. Müstecaplıoğlu, and K. Güven, Phys. Rev. A 84, 033805 (2011).

[9] I. Marino, S. Raghavan, S. Fantoni, S. R. Shenoy, and A. Smerzi, Phys. Rev. A 60, 487 (1999).

[10] A. Smerzi, S. Fantoni, S. Giovanazzi, and S. R. Shenoy, Phys. Rev. Lett. 79, 4950 (1997).

[11] S. Giovanazzi, A. Smerzi, and S. Fantoni, Phys. Rev. Lett. 84, 4521 (2000).

[12] S. Raghavan, A. Smerzi, S. Fantoni, and S. R. Shenoy, Phys. Rev. A 59, 620 (1999).

[13] C. Milian, D. E. Ceballos-Herrera, D. V. Skyrabin, and A. Ferrando, Opt. Lett. 37, 4221 (2012).
[14] C. Lee, W. Hai, L. Shi, X. Zhu, and K. Gao, Phys. Rev. A 64, 053604 (2001).

[15] J. Williams, R. Walser, J. Cooper, E. Cornell, and M. Holland, Phys. Rev. A 59, R31 (1999).

[16] S. H. Strogatz, Nonlinear Dynamics and Chaos with Applications to Physics, Biology, Chemistry and Engineering (Perseus, Cambridge, MA, 1994).

[17] MATCONT is a MATLAB software project for the numerical continuation and bifurcation study of continuous and discrete parametrized dynamical systems developed under the supervision of W. Govaerts and Y. A. Kuznetsov http://sourceforge.net/projects/matcont/

[18] J. Hale and H. Koçak, Dynamics and Bifurcations (SpringerVerlag, New York, 1991).

[19] D. K. Arrowsmith and C. M. Place, Dynamical Systems Differential Equations, Maps and Chaotic Behavior (Chapman \& Hall, Boca Raton, FL, 1992).

[20] A. T. Kanamori and K. Kubodera, IEEE J. Quantum Electron. 29, 2325 (1993)

[21] M. Chauvet, G. Fanjoux, K. P. Huy, V. Nazabal, F. Charpentier, T. Billeton, G. Boudebs, M. Cathelinaud, and S. P. Gorza, Opt. Lett. 34, 1804 (2009).

[22] T. Kaczmarek, Adv. Electr. Telecomm. 1, 59 (2010).

[23] G. P. Agrawal, Nonlinear Fiber Optics, 4th ed. (Elsevier, San Diego, 2007). 